\title{
Uniform spectral properties of one-dimensional quasicrystals, I. Absence of eigenvalues
}

\author{
David Damanik and Daniel Lenz \\ Fachbereich Mathematik \\ Johann Wolfgang Goethe-Universität \\ 60054 Frankfurt/Main \\ Germany
}

May 23, 2022

\begin{abstract}
We consider discrete one-dimensional Schrödinger operators with Sturmian potentials. For a fullmeasure set of rotation numbers including the Fibonacci case we prove absence of eigenvalues for all elements in the hull.
\end{abstract}

\section{Introduction}

In this paper we consider discrete one-dimensional Schrödinger operators in $l^{2}(\mathbf{Z})$ with Sturmian potentials, namely,

$$
\left(H_{\lambda, \alpha, \theta} u\right)(n)=u(n+1)+u(n-1)+\lambda v_{\alpha, \theta}(n) u(n),
$$

where

$$
v_{\alpha, \theta}(n)=\chi_{[1-\alpha, 1)}(n \alpha+\theta \bmod 1),
$$

$\lambda \neq 0, \alpha \in(0,1)$ irrational and $\theta \in[0,1)$, along with the corresponding difference equation

$$
H_{\lambda, \alpha, \theta} u=E u .
$$

The operator family (11) describes a standard one-dimensional quasicrystal model [29, 32] and has been studied in many papers, e.g. [3, , $1,8,21,23$, 31]. Moreover, the operators $H_{\lambda, \alpha, \theta}$ have attracted attention since they exhibit spectral properties such as zero-measure spectrum and purely singular continuous spectral measures that seemingly hold for the entire family in contrast to the almost Mathieu operator where similar properties were shown to hold for a strict subclass of parameter values [22, 26].

To put our present study into perspective, let us consider the class of discrete one-dimensional Schrödinger operators with strictly ergodic (i.e., minimal and uniquely ergodic), aperiodic potentials taking finitely many values. Among these, potentials generated by primitive substitutions and circle maps have received particular interest 11, 2, 6, 7, 9, 10, 13, 14, 18, 19, 20, 23, 33, 34. Sturmian potentials (2) are circle map potentials sharing some crucial properties with potentials generated by primitive substitutions. The general belief is that, as a rule, the spectrum has zero Lebesgue measure and the spectral measures are purely singular continuous. By results of Kotani [25] and Last-Simon [27, absence of absolutely continuous spectrum holds in full generality, that is, for every such family of operators and for every element of the family. Zero-measure 
spectrum was proven for all Sturmian potentials by Bellissard et al. [3] and for a large class of primitive substitutions by Bovier-Ghez [6, 7]. Again, the zero-measure property holds for all elements of the family since, by minimality, the spectrum is constant over the hull. On the other hand, absence of point spectrum has not yet been shown to hold in similar generality. Generic absence of eigenvalues for certain models was proven in 11, 3, 6, 7, 8, 14, 19, 33, while the works 19, 10, 13, 23] contain almost sure results. However, no uniform result, that is, absence of eigenvalues for an entire such family, was known yet. For the particular case of Sturmian potentials, generic absence of eigenvalues is essentially due to Bellissard et al. [3] (the paper does not state the result, see [8, 19] for the result and proofs), whereas almost sure absence of eigenvalues was shown by Kaminaga [23], extending an argument of Delyon-Petritis [13] who had already obtained a partial result.

Our purpose here is to prove the following theorem.

Theorem 1 Suppose $\lim \sup a_{n} \neq 2$, where the $a_{n}$ are the coefficients in the continued fraction expansion of $\alpha$. Then, for every $\lambda$ and every $\theta$, the operator $H_{\lambda, \alpha, \theta}$ has empty point spectrum.

Remarks.

1. The set of $\alpha$ 's obeying the assumption of Theorem 1 has full Lebesgue measure 24].

2. In particular, the Fibonacci case $\alpha=\frac{\sqrt{5}-1}{2}$ is included as all the $a_{n}$ are equal to 1 in this case.

3. Some works associate a slightly different family to the parameters $\lambda, \alpha$ 18, 19] which is larger than the family parametrized by $\theta \in[0,1)$. The proof also works for the additional elements in that larger hull.

4. Our approach is based upon the two-block method [16, 33] and yields additional information about stability properties that will be discussed within a more general context in [12].

5. Another key ingredient in our proof is an analogue to the hierarchical structures and the concept of (de-)composition in self-similar tilings [17]. This will be further exploited in [11].

Thus, combining Theorem 1 with the results of Bellissard et al. [3], the general picture proves to be correct for most parameter values.

Corollary 1 Suppose $\alpha$ obeys the assumption of Theorem 1 . Then, for every $\lambda$ and every $\theta$, the operator $H_{\lambda, \alpha, \theta}$ has purely singular continuous spectrum supported on a Cantor set of zero Lebesgue measure.

The organization of the paper is as follows. Section 2 recalls basic properties of Sturmian potentials as well as the two-block method. Hierarchical structures in Sturmian sequences are studied in Section 3, while Section 4 provides a proof of Theorem 1 .

\section{Basic properties of Sturmian potentials}

Since our argument will be based upon the two-block method, our strategy will be to exhibit appropriate squares adjacent to a certain site $i \in \mathbf{Z}$. This approach is entirely independent of the actual numerical values the potential takes, and we can, without loss of generality, restrict our attention to the particular case $\lambda=1$. That is, we shall study the combinatorial properties of the sequences $v_{\alpha, \theta}$. Consider such a sequence as a two-sided infinite word over the alphabet $A \equiv\{0,1\}$. All these words share the property that their subword complexity is minimal among the aperiodic sequences. Infinite words with this property are called Sturmian. We shall recall some basic facts about these words that will be used in Sections 3 and 4. The material is taken from [3, 5]. Moreover, as a general reference on Sturmian words we want to mention the forthcoming monograph [28]. 
Fix $\alpha$ and consider its continued fraction expansion (for general information on continued fractions see, e.g., [24),

$$
\alpha=\frac{1}{a_{1}+\frac{1}{a_{2}+\frac{1}{a_{3}+\cdots}}} \equiv\left[a_{1}, a_{2}, a_{3}, \ldots\right]
$$

with uniquely determined $a_{n} \in \mathbf{N}$. The associated rational approximants $\frac{p_{n}}{q_{n}}$ obey

$$
\begin{aligned}
& p_{0}=0, p_{1}=1, p_{n}=a_{n} p_{n-1}+p_{n-2}, \\
& q_{0}=1, q_{1}=a_{1}, q_{n}=a_{n} q_{n-1}+q_{n-2} .
\end{aligned}
$$

Define the words $s_{n}$ over the alphabet $A$ by

$$
s_{-1} \equiv 1, \quad s_{0} \equiv 0, \quad s_{1} \equiv s_{0}^{a_{1}-1} s_{-1}, \quad s_{n} \equiv s_{n-1}^{a_{n}} s_{n-2}, n \geq 2 .
$$

In particular, the length of the word $s_{n}$ is equal to $q_{n}, n \geq 0$.

Proposition 2.1 There exist palindromes $\pi_{n}, n \geq 2$, such that

$$
\begin{gathered}
s_{2 n}=\pi_{2 n} 10, \\
s_{2 n+1}=\pi_{2 n+1} 01
\end{gathered}
$$

Proof. See [5], where also the recursive relations obeyed by the $\pi_{n}$ can be found.

By definition, for $n \geq 2, s_{n-1}$ is a prefix of $s_{n}$. Therefore, the following ("right"-) limit exists in an obvious sense,

$$
c_{\alpha} \equiv \lim _{n \rightarrow \infty} s_{n}
$$

Similarly, from (5) we infer that, for $n \geq 1, s_{2 n-2}$ is a suffix of $s_{2 n}$, and hence, the following ("left"-) limit exists,

$$
d_{\alpha} \equiv \lim _{n \rightarrow \infty} s_{2 n}
$$

The exceptional role played by the sequence $v_{\alpha, 0}$ is demonstrated by the following proposition.

Proposition $2.2 v_{\alpha, 0}$ restricted to $\{1,2,3, \ldots\}$ coincides with $c_{\alpha}, v_{\alpha, 0}$ restricted to $\{\ldots,-2,-1,0\}$ coincides with $d_{\alpha}$.

Proof. The first claim was shown by Bellissard et al. in [3]. The second claim follows from the first combined with Proposition 2.1 and the symmetry $v_{\alpha, 0}(-k)=v_{\alpha, 0}(k-1), k \geq 2$, also shown in [3].

For later use, we also want to note the following elementary formula.

Proposition 2.3 For each $n \geq 2, s_{n} s_{n+1}=s_{n+1} s_{n-1}^{a_{n}-1} s_{n-2} s_{n-1}$. 
Proof. $s_{n} s_{n+1}=s_{n} s_{n}^{a_{n+1}} s_{n-1}=s_{n}^{a_{n+1}} s_{n} s_{n-1}=s_{n}^{a_{n+1}} s_{n-1}^{a_{n}} s_{n-2} s_{n-1}=s_{n+1} s_{n-1}^{a_{n}-1} s_{n-2} s_{n-1}$.

The point is that the word $s_{n} s_{n+1}$ has $s_{n+1}$ as a prefix. Note that the dependence of $a_{n}, p_{n}, q_{n}, s_{n}, \pi_{n}$ on $\alpha$ is left implicit. This, however, should not lead to any real confusion as $\alpha$ will always be fixed within a local context.

We now turn to the study of generalized eigenfunctions, i.e. solutions of (3). Recall the standard reformulation of (3) in terms of transfer matrices, namely,

$$
U(n+1)=T_{\lambda, \alpha, \theta}(n, E) U(n),
$$

where $u$ is a solution of (3),

$$
U(n) \equiv\left(\begin{array}{c}
u(n) \\
u(n-1)
\end{array}\right)
$$

and

$$
T_{\lambda, \alpha, \theta}(n, E) \equiv\left(\begin{array}{cc}
E-\lambda v_{\alpha, \theta}(n) & -1 \\
1 & 0
\end{array}\right) .
$$

Thus, the sequence $(u(n))_{n \in \mathbf{Z}}$ is determined by two consecutive values, say $u(0)$ and $u(1)$, and all other values can be determined by applying a matrix product of the form $M_{\lambda, \alpha, \theta}(n, E) \equiv T_{\lambda, \alpha, \theta}(n, E) \times \cdots \times T_{\lambda, \alpha, \theta}(1, E)$ to the vector $U(1)$ (for $n \geq 1$, the case $n \leq 0$ is similar). Note that $\operatorname{det}\left(M_{\lambda, \alpha, \theta}(n, E)\right)=1$. Hence, the characteristic equation of $M_{\lambda, \alpha, \theta}(n, E)$ takes the form

$$
M_{\lambda, \alpha, \theta}(n, E)^{2}-\operatorname{tr}\left(M_{\lambda, \alpha, \theta}(n, E)\right) M_{\lambda, \alpha, \theta}(n, E)+I=0 .
$$

Now, the spectrum of $H_{\lambda, \alpha, \theta}$ is independent of $\theta$ [3] and can thus be denoted by $\Sigma_{\lambda, \alpha}$.

Proposition 2.4 For every $\lambda \neq 0$, there exists $C_{\lambda}$ such that, for every irrational $\alpha$, every $E \in \Sigma_{\lambda, \alpha}$ and every $n \in \mathbf{N}$, we have $\mid \operatorname{tr}\left(M_{\lambda, \alpha, 0}\left(q_{n}, E\right) \mid<C_{\lambda}\right.$.

Proof. See [3].

Before we formulate our basic criterion for absence of eigenvalues, we introduce the following two concepts. Let $v$ be a two-sided sequence over $A$ (think of $v=v_{\alpha, \theta}$ ). A subword $x=x_{1} \ldots x_{l}$ of $v$ is called adjacent to $i \in \mathbf{Z}$ if $v_{i} \ldots v_{i+l-1}=x$ or $v_{i-l+1} \ldots v_{i}=x$. Two finite words $x, y$ having the same length are called conjugate if $x$ is a subword of $y y$. This notion is easily seen to induce an equivalence relation on $A^{l}$, for any fixed $l$. Intuitively, the equivalence class of a word $x=x_{1} \ldots x_{l}$ is given by the collection of all cyclic permutations $x_{j+1} \ldots x_{l} x_{1} \ldots x_{j}$ of $x$.

Lemma 2.5 Suppose $\alpha, \theta$ are such that $v_{\alpha, \theta}$ has infinitely many squares $u_{k} u_{k}$ adjacent to some $i \in \mathbf{Z}$, where $u_{k}$ is conjugate to some $s_{n_{k}}$. Then, for every $\lambda, \sigma_{p p}\left(H_{\lambda, \alpha, \theta}\right)=\emptyset$.

Proof. In case $i=1$, the assertion follows by standard arguments 16, 33 from (10) together with Proposition 2.4. The general case can be reduced to this case by a suitably chosen shift of the sequence $v_{\alpha, \theta}$, which, of course, leaves the spectral type of the associated operators $H_{\lambda, \alpha, \theta}$ invariant.

We close this section by introducing the shift operator $T$ on functions on $\mathbf{Z}$, i.e.

$$
(T f)(n) \equiv f(n+1)
$$

for arbitrary functions $f$ on $\mathbf{Z}$. 


\section{The partition lemma}

Consider for a fixed $\alpha$ the family (in $\theta$ ) of all the sequences of the form $\left(v_{\alpha, \theta}(k)\right)_{k \in \mathbf{Z}}$. It is well known that in each sequence of the family the same words occur. Moreover any of these words occurs with a fixed frequency greater than zero which is independent of the sequence (cf. Appendix of [18]). Thus, from a measure theoretical point of view, the family $\left\{\left(v_{\alpha, \theta}(k)\right)_{k \in \mathbf{Z}} \mid \theta \in[0,1)\right\}$ behaves very uniformly. However, to prove the absence of eigenvalues for all $\theta$ we need a kind of uniform topological structure. In this section we provide this structure by showing that each sequence $\left(v_{\alpha, \theta}(k)\right)_{k \in \mathbf{Z}}$ can be decomposed into blocks of the form $s_{n}$ and $s_{n-1}$ for all $n \in \mathbf{N}_{\mathbf{0}}$. Here we denote by $\mathbf{N}_{\mathbf{0}}$ the set of all integers together with 0, i.e. $\mathbf{N}_{\mathbf{0}}=\{\mathbf{0}, \mathbf{1}, \mathbf{2}, \ldots\}$.

Definition 1 Let $n \in \mathbf{N}_{\mathbf{0}}$ be given. An $(n, \alpha)$-partition of a sequence $\left(f_{k}\right)_{k \in \mathbf{Z}}$ with $f_{k} \in\{0,1\}$ is a sequence $\left(I_{j}, z_{j}\right)_{j \in \mathbf{Z}}$ of pairs $I_{j}=\left\{d_{j}, d_{j}+1, \ldots, d_{j+1}-1\right\} \subset \mathbf{Z}$ and $z_{j} \in\left\{s_{n}, s_{n-1}\right\}$ with $0 \in I_{0}$ s.t.

$$
f_{d_{j}} f_{d_{j}+1} \ldots f_{d_{j+1}-1}=z_{j}
$$

for all $j \in \mathbf{Z}$.

An $(n, \alpha)$-partition of a function $f: \mathbf{Z} \longrightarrow\{\mathbf{0}, \mathbf{1}\}$ is an $(n, \alpha)$-partition of the sequence $(f(n))_{n \in \mathbf{Z}}$.

The $z_{j}$ are referred to as blocks in the n-partition or more specifically as blocks of the form $s_{n}$ if $z_{j}=s_{n}$, and as blocks of the form $s_{n-1}$ if $z_{j}=s_{n-1}$. The $I_{j}$ are referred to as positions of the blocks $z_{j}$.

In the sequel we will sometimes suppress the dependence on $\alpha$ if it is clear from the context to which $\alpha$ we refer. In particular, we will write $n$-partition instead of $(n, \alpha)$-partition.

Remarks.

1. One can think of an $n$-partition as a tiling of $\mathbf{Z}$ by $s_{n}$ and $s_{n-1}$ generating $f$. So $f$ is composed out of the blocks $z_{j}$ at the positions $I_{j}$.

2. Our notion of $n$-partition is analogous to the notion of $n$th composition used in the study of self similar tilings [30]. This notion has been used by Hof in [18]. There he studies the Lyapunov exponent and the integrated density of states of discrete Schrödinger operators with a potential generated by a primitive substitution.

As $s_{0}=0$ and $s_{-1}=1$ every $f: \mathbf{Z} \longrightarrow\{\mathbf{0}, \mathbf{1}\}$ admits a 0 -partition. But for a general $f$ there does not necessarily exist an $n$-partition for $n>0$. However, it is crucial to our analysis of the eigenvalue problem for $H_{\lambda, \alpha, \theta}$ that for the sequences $v_{\alpha, \theta}$ there do exist unique $n$-partitions for all $n \in \mathbf{N}_{\mathbf{0}}$. This is the content of the next lemma.

Lemma 3.1 (i) For every $n \in \mathbf{N}_{\mathbf{0}}$, there exists a unique $n$-partition of $v_{\alpha, 0}$.

(ii) For every $n \in \mathbf{N}_{\mathbf{0}}$ and every $\theta \in[0,1)$, there exists a unique $n$-partition of $v_{\alpha, \theta}$.

Proof. Let $\alpha=\left[a_{1}, a_{2}, \ldots\right]$ be the continued fraction expansion of $\alpha$ (cf. equation 4 ).

(i) Existence: Set $v \equiv v_{\alpha, 0}$. We show that there are $n$-partitions of $(v(k))_{k \geq 1}$ and of $(v(k))_{k<0}$. Here an $n$-partition of a one sided sequence is defined in the obvious way. By (5), (8) and Proposition 2.2, it is clear that there exists an $n$-partition of $(v(k))_{k \geq 1}$ for all $n$.

The existence of an $n$-partition for $(v(k))_{k \leq 0}$ follows similarly by (5), (9) and Proposition 2.2 .

Uniqueness: This follows by induction: As $s_{0}=0, s_{-1}=1$ uniqueness is clear for $n=0$. By (5), every $(n+1)$-partition gives rise to an $n$-partition and the positions of the $s_{n+1}$ in the $(n+1)$-partition are determined by the positions of $s_{n-1}$ in the $n$-partition. Thus, uniqueness of the $n$-partition implies uniqueness of the $(n+1)$-partition.

(ii) Fix $\theta \in[0,1)$. As $\alpha$ is irrational there exists a sequence $\left(n_{k}\right)_{k \in \mathbf{N}}, n_{k} \in \mathbf{N}$, s.t. the sequence $\left(T^{n_{k}} v_{\alpha, 0}\right)_{k \in \mathbf{N}}$ 
converges to $v_{\alpha, \theta}$ in the product topology on $\{0,1\}^{\mathbf{Z}}$ for $k \rightarrow \infty$. By (i), it is clear that the $T^{n_{k}} v_{\alpha, 0}$ admit unique $n$-partitions for all $n \in \mathbf{N}_{\mathbf{0}}$. To use this to conclude (ii) we introduce the following notion of convergence:

Let $f_{k}, k \in \mathbf{N}$, and $f$ be functions for which there exist unique $n$-partitions denoted by $\left(I_{j}^{k}, z_{j}^{k}\right)$ and $\left(I_{j}, z_{j}\right)$, respectively. We say that the $f_{k}$ converge to $f$ in the $n$-sense for $k \rightarrow \infty$ if for all $C>0$ there exists a $k_{0}$ s.t. for $k \geq k_{0}$

$$
\left(I_{j}^{k}, z_{j}^{k}\right)=\left(I_{j}, z_{j}\right) \text { for all } I_{j} \subset(-C, C)
$$

holds.

Clearly, (ii) follows if we prove the following claim.

Claim. For each $n$ there exists a unique $n$-partition of $v_{\alpha, \theta}$ and the sequence $\left(T^{n_{k}} v_{\alpha, 0}\right)_{k \in \mathbf{N}_{\mathbf{o}}}$ converges to $v_{\alpha, \theta}$ in the $n$-sense for $k \rightarrow \infty$.

Proof of the claim. This will be done by induction. We will consider two cases.

Case 1: $a_{1}=1$.

As $s_{-1}=s_{1}=1$ and $s_{0}=0$, the cases $n=0$ and $n=1$ are clear. So suppose the statement is true for $n \geq 1$ fixed. Let $\left(I_{j}, z_{j}\right)$ be the $n$-partition of $v_{\alpha, \theta}$. By $s_{n+1}=s_{n}^{a_{n+1}} s_{n-1}$ (cf. equation 5), the existence of an $(n+1)$-partition of $v_{\alpha, \theta}$ will follow if we show that to the left of each block of the form $s_{n-1}$ in the $n$-partition of $v_{\alpha, \theta}$ there are at least $a_{n+1}$ blocks $s_{n}$. That is, we have to show that $z_{j}=s_{n-1}$ for $j \in \mathbf{Z}$ implies $z_{k}=s_{n}$ for $k=j-a_{n+1}, \ldots, j-1$. As $T^{n_{k}} v_{\alpha, 0}$ admits a unique $n$-partition for each $n \in \mathbf{N}_{\mathbf{0}}$, there are at least $a_{n+1}$ blocks $s_{n}$ to the left of each block of the form $s_{n-1}$ in the $n$-partition of $T^{n_{k}} v_{\alpha, 0}$. As the $T^{n_{k}} v_{\alpha, 0}$ converge to $v_{\alpha, \theta}$ in the $n$-sense, the corresponding statement is true for $v_{\alpha, \theta}$. This gives the existence of an $(n+1)$-partition of $v_{\alpha, \theta}$. The uniqueness of the $(n+1)$-partition follows from the uniqueness of the $n$-partition as in $(i)$. As the blocks $s_{n+1}$ in the $(n+1)$-partition of $v_{\alpha, \theta}$ arise from blocks $s_{n}^{a_{n+1}} s_{n-1}$ in the $n$-partition of $v_{\alpha, \theta}$, it is clear that the convergence of $T^{n_{k}} v_{\alpha, 0}$ to $v_{\alpha, \theta}$ in the $n$-sense implies the convergence in the $(n+1)$-sense. This proves the claim in Case 1 .

Case 2: $a_{1}>1$.

As $s_{-1}=1$ and $s_{0}=0$, the case $n=0$ is clear. So fix $n \geq 0$. If $n>0$ we can continue exactly as in Case 1. If $n=0$ we can continue as in Case 1 after replacing $a_{n+1}=a_{1}$ by $a_{1}-1 \geq 1$. This proves the claim in Case 2.

The proof of the lemma is therefore finished.

Corollary 2 Let, for $n \in \mathbf{N},\left(I_{j}^{n}, z_{j}^{n}\right)$ be the n-partition of $v_{\alpha, \theta}$. If $z_{j}^{n}=s_{n-1}$, then $z_{j-1}^{n}=z_{j+1}^{n}=s_{n}$. If $z_{j}^{n}=s_{n}$, then there is an interval $I=\{d, d+1, \ldots, d+l-1\} \subset \mathbf{Z}$ of length $l \in\left\{a_{n+1}, a_{n+1}+1\right\}$ with $j \in I$ and $z_{i}^{n}=s_{n}$ for all $i \in I$ and $z_{d-1}^{n}=z_{d+l}^{n}=s_{n-1}$.

Proof. By the existence part of the partition lemma, there exists an $(n+1)$-partition of $v_{\alpha, \theta}$. By the uniqueness part of the partition lemma and the formula $s_{n+1}=s_{n}^{a_{n+1}} s_{n-1}$, all the blocks of the form $s_{n-1}$ in the $n$-partition of $v_{\alpha, \theta}$ arise from blocks of the form $s_{n+1}$ in the $(n+1)$-partition. This shows that there is no $j \in \mathbf{Z}$ with $z_{j}^{n}=z_{j+1}^{n}=s_{n-1}$ and that there are at least $a_{n+1}$ blocks of the form $s_{n}$ between two blocks of the form $s_{n-1}$. That there are at most $a_{n+1}+1$ such blocks follows, as there are not two adjacent blocks of the form $s_{n}$ in the $(n+1)$-partition. This proves the corollary.

\section{Remarks.}

1. Define $\Omega$ to be the set of accumulation points of translates of $v_{\alpha, 0}$ with respect to pointwise convergence, that is,

$$
\Omega(\alpha) \equiv\left\{\omega \in A^{\mathbf{z}} \mid \omega=\lim T^{n_{i}} v_{\alpha, 0}, n_{i} \rightarrow \infty\right\} .
$$

Then the method of the previous lemma can easily be adopted to prove the existence of a unique $n$-partition for all $\omega \in \Omega(\alpha)$. We refer the reader to [12] for a discussion of the relationship between $\Omega(\alpha)$ and the set $\left\{v_{\alpha, \theta} \mid \theta \in[0,1)\right\}$. 
2. The above lemma and a careful analysis of $v_{\alpha, 0}$ allow to show that the blocks $s_{n}$ and $s_{n-1}$ in the $n$-partition of an $\omega \in \Omega(\alpha)$ occur with fixed frequency, see [11] for details. This can be used together with Theorem 1 of $\left[15\right.$ to show that indeed every word that occurs in some $\omega_{0} \in \Omega(\alpha)$ occurs in every $\omega \in \Omega(\alpha)$ with a fixed frequency greater than zero. Thus, the above partition lemma is indeed a stronger result than the results about the frequencies of words mentioned at the beginning of this section.

\section{Absence of eigenvalues}

Let $\alpha=\left[a_{1}, a_{2}, \ldots\right]$ be the continued fraction expansion of $\alpha$ (cf. equation 4 ).

The proof of Theorem 1 will be split into two parts.

Proposition 4.1 If $\lim \sup _{n \rightarrow \infty} a_{n} \geq 3$, then, for every $\theta$ and every $\lambda$, the operator $H_{\lambda, \alpha, \theta}$ has no eigenvalues.

Proposition 4.2 If $\lim \sup _{n \rightarrow \infty} a_{n}=1$, then, for every $\theta$ and every $\lambda$, the operator $H_{\lambda, \alpha, \theta}$ has no eigenvalues.

Remark. In fact the proofs yield the absence of eigenvalues for all potentials in the respective hulls

$$
\Omega(\lambda, \alpha):=\{\lambda \omega: \omega \in \Omega(\alpha)\} .
$$

Proof of Proposition 4.1. Fix $\theta \in[0,1)$. We will exclude eigenvalues of $H_{\lambda, \alpha, \theta}$ using Lemma 2.5, that is, by exhibiting many appropriate squares in $v_{\alpha, \theta}$ at zero. By the partition lemma 3.1, there is for each $n \in \mathbf{N}_{\mathbf{0}}$ an $n$-partition

$$
\left(\left(I_{j}^{n}, z_{j}^{n}\right)\right)_{j \in \mathbf{Z}}, I_{j}^{n}=\left\{d_{j}^{n}, \ldots, d_{j+1}^{n}-1\right\}, z_{j}^{n} \in\left\{s_{n}, s_{n-1}\right\}
$$

of $v_{\alpha, \theta}$. We will consider two cases.

Case 1: There are infinitely many $n \in \mathbf{N}_{\mathbf{0}}$ with $z_{0}^{n}=s_{n-1}$.

Consider such an $n$ with $n \geq 4$. Corollary 2 yields $z_{1}^{n}=s_{n}$. As $s_{n-1}$ is a prefix of $s_{n}$ and $z_{2}^{n} \in\left\{s_{n}, s_{n-1}\right\}$ we have

$$
z_{0}^{n} z_{1}^{n} z_{2}^{n}=s_{n-1} s_{n} s_{n-1} w
$$

with a suitable word $w$. Using $s_{n}=s_{n-1}^{a_{n}} s_{n-2}$ (cf. equation 5), we get

$$
z_{0}^{n} z_{1}^{n} z_{2}^{n}=s_{n-1} s_{n-1}^{a_{n}} s_{n-2} s_{n-1} w .
$$

By $s_{n-2} s_{n-1}=s_{n-1} v$ with a suitable $v$ (cf. Proposition 2.3), we finally arrive at

$$
z_{0}^{n} z_{1}^{n} z_{2}^{n}=s_{n-1} s_{n-1}^{a_{n}} s_{n-1} v w .
$$

This implies

$$
v_{\alpha, \theta}\left(d_{0}^{n}\right) v_{\alpha, \theta}\left(d_{0}^{n}+1\right) \ldots v_{\alpha, \theta}\left(d_{0}^{n}+3\left|s_{n-1}\right|-1\right)=s_{n-1} s_{n-1} s_{n-1},
$$

where $0 \in\left\{d_{0}^{n}, \ldots, d_{0}^{n}+\left|s_{n-1}\right|-1\right)$. Thus, there exists a square $x x$ with $x$ being a cyclic permutation of $s_{n-1}$ to the right of zero. Since this is true for infinitely many $n$, we can use Lemma 2.5 to exclude eigenvalues.

Case 2: There is an $n_{0} \in \mathbf{N}_{\mathbf{0}}$ s.t. $z_{0}^{n}=s_{n}$ for all $n \geq n_{0}$

By $\lim \sup _{n \rightarrow \infty} a_{n} \geq 3$, there are infinitely many $n \geq n_{0}$ s.t. $a_{n} \geq 3$. Fix such an $n$. As $a_{n} \geq 3$ and $z_{0}^{n}=s_{n}$, there are three cases by Corollary 2 .

Subcase 1: $z_{0}^{n}=z_{1}^{n}=z_{2}^{n}=s_{n}$. 
In this case we have $z_{0}^{n} z_{1}^{n} z_{2}^{n}=s_{n} s_{n} s_{n}$.

Subcase 2: $z_{0}^{n}=z_{-1}^{n}=z_{-2}^{n}=s_{n}$.

In this case we have $z_{-2}^{n} z_{-1}^{n} z_{0}^{n}=s_{n} s_{n} s_{n}$.

Subcase 3: $z_{-1}^{n}=z_{0}^{n}=z_{1}^{n}=s_{n}, z_{-2}^{n}=z_{2}^{n}=s_{n-1}$.

Calculating as in Case 1 we get $z_{0}^{n} z_{1}^{n} z_{2}^{n} z_{3}^{n}=s_{n} s_{n} s_{n} w$ with a suitable word $w$.

Thus, in all subcases we can conclude as in Case 1 that there exists a square $x x$, with $x$ being a cyclic permutation of $s_{n}$ either to the left or to the right of zero. Since this applies to infinitely many $n$, we can use Lemma 2.5 to exclude eigenvalues in Case 2 as well. This proves the proposition.

Proof of Proposition 4.2. The proof is similar to the proof of Proposition 4.1. So fix $\theta \in[0,1)$. By Lemma 3.1, there exists a unique $n$-partition $\left(\left(I_{j}^{n}, z_{j}^{n}\right)\right)_{j \in \mathbf{Z}}$ of $v_{\alpha, \theta}$. Again we will consider two cases.

Case 1: There are infinitely many $n \in \mathbf{N}_{\mathbf{0}}$ with $z_{0}^{n}=s_{n-1}$.

This case can be treated as in the proof of Theorem 4.1.

Case 2: There is an $n_{0} \in \mathbf{N}_{\mathbf{0}}$ s.t. $z_{0}^{n}=s_{n}$ for all $n \geq n_{0}$.

By $\lim \sup _{n \rightarrow \infty} a_{n}=1$, there is an $n_{1} \in \mathbf{N}_{\mathbf{0}}$ s.t. $a_{n}=1$ for all $n \geq n_{1}$. Let $c:=\max \left\{n_{0}, n_{1}\right\}$.

As $z_{0}^{n}=s_{n}$ and $s_{n+1}=s_{n} s_{n-1}$ for all $n \geq c$, it follows easily by induction that

$$
d_{0}^{n}=d_{0}^{c} \text { for all } n \geq c .
$$

Moreover $z_{0}^{n}=s_{n}$ and $s_{n+1}=s_{n} s_{n-1}$ imply $z_{1}^{n}=s_{n-1}$ for all $n \geq c$ and this in turn implies $z_{2}^{n}=s_{n}$ for all $n \geq c$. We therefore have

$$
z_{0}^{n} z_{1}^{n} z_{2}^{n}=s_{n} s_{n-1} s_{n}=s_{n} s_{n} w
$$

where we used $s_{n-1} s_{n}=s_{n} w$ with a suitable word $w$ (cf. Proposition 2.3). Putting all this together, we see

$$
v_{\alpha, \theta}\left(d_{0}^{c}\right) \ldots v_{\alpha, \theta}\left(d_{0}^{c}+2\left|s_{n}\right|-1\right)=s_{n} s_{n} \text { for all } n \geq c .
$$

Again, an application of Lemma 2.5 yields the desired absence of eigenvalues, concluding the proof.

Acknowledgment. One of us (D. L.) gratefully acknowledges financial support from Studienstiftung des deutschen Volkes (Doktorandenstipendium).

\section{References}

[1] Bellissard, J., Bovier, A., Ghez, J.-M. : Spectral properties of a tight binding Hamiltonian with period doubling potential, Commun. Math. Phys. 135, 379-399 (1991)

[2] Bellissard, J., Bovier, A., Ghez, J.-M. : Gap labelling theorems for one-dimensional discrete Schrödinger operators, Rev. Math. Phys. 4, 1-37 (1992)

[3] Bellissard, J., Iochum, B., Scoppola, E., Testard, D. : Spectral properties of one-dimensional quasicrystals, Commun. Math. Phys. 125, 527-543 (1989)

[4] Bellissard, J., Iochum, B., Testard, D. : Continuity properties of the electronic spectrum of 1D quasicrystals, Commun. Math. Phys. 141, 353-380 (1991)

[5] Berstel, J. : Recent results in Sturmian words, in Dassow, J. and Salomaa, A. (Eds.), Developments in Language Theory, World Scientific, 13-24 (1996)

[6] Bovier, A., Ghez, J.-M. : Spectral properties of one-dimensional Schrödinger operators with potentials generated by substitutions, Commun. Math. Phys. 158, 45-66 (1993) 
[7] Bovier, A., Ghez, J.-M. : Erratum. Spectral properties of one-dimensional Schrödinger operators with potentials generated by substitutions, Commun. Math. Phys. 166, 431-432 (1994)

[8] Damanik, D. : $\alpha$-continuity properties of one-dimensional quasicrystals, Commun. Math. Phys. 192, 169-182 (1998)

[9] Damanik, D. : Singular continuous spectrum for the period doubling Hamiltonian on a set of full measure, Commun. Math. Phys. 196, 477-483 (1998)

[10] Damanik, D. : Singular continuous spectrum for a class of substitution Hamiltonians, Lett. Math. Phys., to appear

[11] Damanik, D., Lenz, D. : Uniform spectral properties of one-dimensional quasicrystals, II. The Lyapunov exponent, in preparation

[12] Damanik, D., Lenz, D. : Half-line eigenfunction estimates and stability of singular continuous spectrum, in preparation

[13] Delyon, F., Petritis, D. : Absence of localization in a class of Schrödinger operators with quasiperiodic potential, Commun. Math. Phys. 103, 441-444 (1986)

[14] Delyon, F., Peyrière, J. : Recurrence of the eigenstates of a Schrödinger operator with automatic potential, J. Stat. Phys. 64, 363-368 (1991)

[15] Geerse, C., Hof, A. : Lattice gas models on self-similar aperiodic tilings, Rev. Math. Phys. 3, 163-221 (1991)

[16] Gordon, A. : On the point spectrum of the one-dimensional Schrödinger operator, Usp. Math. Nauk 31, $257-258$ (1976)

[17] Grünbaum, B., Shephard, G. C. : Tilings and Patterns, Freeman and Company, New York (1987)

[18] Hof, A. : Some remarks on discrete aperiodic Schrödinger operators, J. Stat. Phys. 72, 1353-1374 (1993)

[19] Hof, A., Knill, O., Simon, B. : Singular continuous spectrum for palindromic Schrödinger operators, Commun. Math. Phys. 174, 149-159 (1995)

[20] Iochum, B., Testard, D. : Power law growth for the resistance in the Fibonacci model, J. Stat. Phys. 65, 715-723 (1991)

[21] Iochum, B., Raymond, L., Testard, D. : Resistance of one-dimensional quasicrystals, Physica A 187, 353-368 (1992)

[22] Jitomirskaya, S. : Almost everything about the almost Mathieu operator, II, Proceedings of XI International Congress of Mathematical Physics, Paris 1994, Int. Press, 373-382 (1995)

[23] Kaminaga, M. : Absence of point spectrum for a class of discrete Schrödinger operators with quasiperiodic potential, Forum Math. 8, 63-69 (1996)

[24] Khintchine, A. : Continued Fractions, Noordhoff, Groningen (1963)

[25] Kotani, S. : Jacobi matrices with random potentials taking finitely many values, Rev. Math. Phys. 1, 129-133 (1989)

[26] Last, Y. : Almost everything about the almost Mathieu operator, I, Proceedings of XI International Congress of Mathematical Physics, Paris 1994, Int. Press, 366-372 (1995)

[27] Last, Y., Simon, B. : Eigenfunctions, transfer matrices, and absolutely continuous spectrum of onedimensional Schrödinger operators, Invent. Math., to appear 
[28] Lothaire, M. : Algebraic combinatorics on words, in preparation

[29] Luck, J., Petritis, D. : Phonon spectra in one-dimensional quasicrystals, J. Stat. Phys. 42, 289-310 (1986)

[30] Lunnon, W., Pleasants, P. : Quasicristallographic tilings, J. Math. Pures et Appl. 66, 217-263 (1987)

[31] Raymond, L. : A constructive gap labelling for the discrete Schrödinger operator on a quasiperiodic chain, preprint

[32] Senechal, M. : Quasicrystals and Geometry, Cambridge University Press (1995)

[33] Sütő, A. : The spectrum of a quasiperiodic Schrödinger operator, Commun. Math. Phys. 111, 409-415 (1987)

[34] Sütö, A. : Singular continuous spectrum on a Cantor set of zero Lebesgue measure for the Fibonacci Hamiltonian, J. Stat. Phys. 56, 525-531 (1989) 